Service social

\title{
Quand le Good Lives Model rencontre les travailleurs psychosociaux : une invitation à un changement de posture professionnelle
}

\section{Serge Corneille et Bérengère Devillers}

Volume 63, numéro 1, 2017

Interventions en matière d'agressions sexuelles

URI : https://id.erudit.org/iderudit/1040027ar

DOI : https://doi.org/10.7202/1040027ar

Aller au sommaire du numéro

Éditeur(s)

École de service social de l’Université Laval

ISSN

1708-1734 (numérique)

Découvrir la revue

Citer cet article

Corneille, S. \& Devillers, B. (2017). Quand le Good Lives Model rencontre les travailleurs psychosociaux : une invitation à un changement de posture professionnelle. Service social, 63(1), 12-28. https://doi.org/10.7202/1040027ar
Résumé de l'article

Rédigé par deux professionnels qui travaillent avec cetteapproche (et ont contribué à la faire connaîtredans la Francophonie), cet article présente les lignesdirectrices et la spécificité du Good Lives Model, enparticulier lorsqu'utilisé par des intervenantspsychosociaux. 


\title{
Quand le Good Lives Model rencontre les travailleurs psychosociaux : une invitation à un changement de posture professionnelle
}

\author{
CORNEILLE, Serge \\ DEVILLERS, Bérengère
}

\section{RÉSUMÉ}

Rédigé par deux professionnels qui travaillent avec cette approche (et ont contribué à la faire connaître dans la Francophonie), cet article présente les lignes directrices et la spécificité du Good Lives Model, en particulier lorsqu'utilisé par des intervenants psychosociaux.

Mots-clés : Good Lives Model, réhabilitation, délinquant, travail social.

\begin{abstract}
Written by two professionals who work in this approach (and helped to make it known in the French-speaking world), this article presents the guidelines and specificity of Good Lives Model, especially when used by psychologists and social workers.
\end{abstract}

Keywords: Good Lives Model, rehabilitation, delinquency, social work.

Le «Good Lives Model » (G.L.M.) est à la base un modèle de réhabilitation des délinquants sexuels (Ward et Beech, 2006) mais il a ensuite été appliqué à d'autres populations délinquantes spécifiques telles que les populations psychiatrique (Barnao, Robertson et Ward, 2010) ou adolescente (Print, 2013). Plus récemment, des applications françaises du G.L.M. s'expérimentent via le programme à dimension culturelle "SERA" en Outre-Mer (Dieu, 2017), via le programme "DEMAIN" pour le Quartier pour Peines Aménagées de Nantes (Dieu, 2016) ou encore le programme "CéSURE" dans les établissements pénitentiaires à destination du public radicalisé (Dieu, 2015) 》. Ces différentes expérimentations sont tirées du Protocole PRATIC de groupe (Dieu, 2017).

Auparavant, les travailleurs sociaux n'étaient guère concernés par les populations délinquantes. En effet, dans un cadre de justice rétributive, nul intérêt n'était accordé au risque de récidive, le justiciable ayant à s'acquitter d'une rétribution, sous forme d'une amende ou d'une peine, pour les crimes et délits pour lesquels il avait été condamné.

Aujourd'hui, nos interventions se situent dans un contexte de justice réparatrice et/ou restaurative. Dans un tel contexte, un accent majeur est mis, d'une part, sur la restauration du lien social et, d'autre part, sur les besoins de la victime. Une place prioritaire va donc être donnée à la problématique de la prévention de la récidive et confiée à de nombreux intervenants dont des travailleurs psychosociaux.

De ce fait, les professionnels de la relation d'aide vont occuper une fonction qui leur est inhabituelle et parfois inconfortable dans la mesure où ils vont devoir mener des actions visant à satisfaire les besoins de tiers (victimes, société) plutôt qu'à satisfaire ceux des usagers eux-mêmes. Au nom des intérêts de la société, la cible unique et finale de 
l'intervention sera la prévention de la récidive, tandis qu'au nom des intérêts des victimes, un certain nombre de cibles intermédiaires seront intégrées dans les programmes d'intervention quand bien même n'auraient-ils pas de lien avéré avec le risque de récidive lui-même (reconnaissance des faits et de la responsabilité de l'auteur, empathie par rapport à la victime, etc.).

Cet article vise à établir en quoi le G.L.M. redéfinit la position du justiciable mais également celle de l'intervenant psychosocial dans les interventions menées auprès de délinquants sexuels, qu'ils soient adolescents ou adultes.

Afin de mettre le G.L.M. en perspective avec les modèles qui l'ont précédé et de pouvoir ainsi mieux cerner son aspect novateur, nous nous intéresserons, dans un premier temps, au modèle de prévention de la récidive (Pithers et al., 1983) qui a été le premier modèle spécifique de traitement des délinquants sexuels.

Dans un second temps, nous verrons quelles sont les composantes d'un programme de réhabilitation. Le G.L.M. sera décrit dans ses principes généraux, objectifs et implications. Nous verrons également comment le G.L.M. offre une opportunité de se situer dans une logique d'accompagnement, axée sur les compétences et les ressources de la personne, en mobilisant directement l'intervenant psychosocial et en le responsabilisant dans le succès de la prise en charge.

Une réflexion sera également menée sur la compatibilité entre les valeurs du G.L.M. et celles habituellement véhiculées dans l'accompagnement psychosocial.

Enfin, une vignette clinique viendra illustrer les caractéristiques qui peuvent se distinguer, d'une part, d'une prise en charge classique pluridisciplinaire et, d'autre part, d'une prise en charge inspirée du G.L.M.

\section{Du modèle de prévention de la récidive au contexte actuel}

Le premier modèle spécifique de traitement des délinquants sexuels a été le modèle de prévention de la récidive (ou de la rechute) s'inspirant du traitement des conduites addictives. Ce modèle visait à éradiquer ou à inhiber (orchidectcastration chimique, satiation masturbatoire) une excitation sexuelle définie comme déviante et à tenter de réorienter l'objet même de cette excitation sexuelle déviante (réorientation fantasmatique). Ce modèle est fondé sur les principes de consentement/résistance à l'intervention. L'attention portée aux besoins de l'usager est peu perceptible. L'usager est considéré comme le récepteur de l'intervention menée, laquelle vise uniquement à la prévention de la récidive. L'intervenant psychosocial y est donc perçu comme le simple administrateur des techniques coercitives visant l'intérêt public. Les cibles de l'intervention sont confinées à des cognitions et comportements dits déviants plutôt que de s'adresser à des justiciables dans leur globalité.

Les traitements issus de ce modèle ont rapidement fait la preuve de leur inefficacité au regard des objectifs qu'ils étaient censés poursuivre.

Apparurent ensuite, dans les années 1990, les principes de Risques-Besoins-Réceptivité plus connus sous leur appellation (R.B.R. en français ou R.N.R. en anglais). Le R.B.R. (Andrews, Bonta et Wormith, 2011) n'est pas à proprement parler un modèle étiologique de la délinquance mais plutôt un ensemble de principes qui, s'ils sont correctement appliqués, rendent les interventions plus efficaces du point de vue de la prévention de la récidive. En effet, les principes du R.B.R. ne se substituent en rien aux fondements du modèle de 
prévention de la rechute mais précisent quelles sont les modalités d'application les plus efficaces de ce modèle.

De fait, les principes R.B.R. vont indéniablement permettre une amélioration significative de l'efficacité des traitements en termes stricts de prévention de la récidive. Les apports majeurs du R.B.R. sont, à nos yeux, une moindre standardisation des interventions et une attention accrue portée à la motivation du justiciable à s'engager dans celles-ci.

Néanmoins, sa limite majeure réside dans le fait que les intérêts du justiciable et les cibles de l'intervention persistent à être présentés comme exclusifs l'un de l'autre et que l'unique cible des interventions se fondant sur les principes R.B.R. reste, sans nuance, la prévention de la récidive. Les principes R.B.R. ne permettent donc pas d'éviter les écueils du modèle de prévention de la rechute: l'intervention est fondée sur des principes de privation, de renoncement et sur la stigmatisation des carences ou des déviances du justiciable. Par ailleurs, le modèle de prévention de la rechute, même agrémenté des principes R.B.R., reste centré sur les cognitions et comportements sexuellement déviants, alors même que les délinquants sexuels, lorsqu'ils récidivent, le font généralement sur un mode non sexuel.

À y regarder de plus près, le modèle R.B.R. offre, certes, des principes directeurs dans le travail avec les délinquants sexuels mais ne donne pas aux intervenants psychosociaux, une manière de dépasser les limites imposées par le cadre d'aide dite contrainte. En effet, une limite reconnue au R.B.R. est de ne pas pouvoir parvenir à motiver et à impliquer les sujets dans la prise en charge. Les principes auxquels renvoie le R.B.R. s'appuient sur la motivation externe (se conformer aux injonctions judiciaires afin d'éviter une nouvelle incarcération) et moins directement sur la création d'une motivation dite interne (p. ex. m'engager dans des conduites déviantes me prive de la présence de mes proches). Le manque de centration sur les éléments constitutifs de la motivation interne, ajouté au fait que les programmes de prévention de la récidive sont davantage centrés sur les besoins de la communauté (diminuer la dangerosité) que sur ceux du participant (qu'en est-il de son bienêtre ?), représentent deux caractéristiques susceptibles de conduire à un taux d'abandon de 30 à $50 \%$, voire $70 \%$, selon les auteurs. Or, il est communément admis que le risque de récidive est plus élevé si on abandonne un traitement que si aucun traitement n'est entamé (Hanson et Morton-Bourgon, 2005).

Ainsi, les critiques formulées au R.B.R. peuvent être résumées comme suit (inspiré de Vanderstukken et Benbouriche, 2013) :

$\checkmark$ un ciblage accru sur la gestion du risque plutôt que sur un objectif de définition d'une vie positive et épanouissante ;

$\checkmark$ un manque de prise en compte des besoins primaires de l'être humain ;

$\checkmark$ une perception du délinquant comme un ensemble de facteurs de risque et non comme un être complexe qui cherche à se donner de la valeur et à trouver un sens à sa vie ;

$\checkmark$ une tendance à intervenir de manière mécanique et négative : on se concentre sur ce qui doit être évité plutôt que sur les compétences à acquérir ;

$\checkmark$ un moindre accent mis sur la construction d'une identité positive et cohérente ;

$\checkmark$ le peu d'attention portée aux besoins non criminogènes alors que ceux-ci favorisent l'alliance thérapeutique et la motivation du sujet à s'investir dans la thérapie 
(Marshall et al., 2001; Mann et al., 2004, cités par Vanderstukken et Benbouriche, 2013) ;

$\checkmark$ ne vise pas à fournir les conditions nécessaires pour vivre une meilleure vie mais à enseigner uniquement comment minimiser ses possibilités de récidiver.

II faudra attendre le début des années 2000 pour que, sous l'impulsion de Tony Ward, se développe le premier modèle alternatif au modèle de prévention de la rechute : le Good Lives Model ou "modèle des vies fondées sur la satisfaction des besoins humains fondamentaux ».

\section{La naissance du modèle G.L.M.}

\section{Qu'entend-on par réhabilitation?}

Wade et de Jong (2000) in Ward et Maruna (2007, p. 5-6, traduit par nos soins) reconnaissent que " une définition de la réhabilitation n'a pas encore trouvé d'accord universel dans la profession médicale ». Selon eux, la définition la plus proche de l'utilisation de ce mot est la suivante : la réhabilitation est un processus réitératif, actif, éducatif, de résolution de problèmes, centré sur les comportements du patient avec les composantes suivantes :

$\checkmark$ l'évaluation : identification de la nature et de l'étendue des problèmes du patient et les facteurs pertinents à leurs résolutions;

$\checkmark$ l'établissement d'objectifs;

$\checkmark$ l'intervention, laquelle peut inclure soit un traitement qui impacte le processus de changement, soit un support qui maintient la qualité de vie du patient et sa sécurité;

$\checkmark$ la vérification des effets de l'intervention.

Dans la perspective G.L.M., une théorie de la réhabilitation devrait posséder les ressources pour guider les praticiens dans tous les aspects de leur travail. Une théorie de la réhabilitation doit notamment pouvoir préciser explicitement les aspects de comportement, relationnel et motivationnel du traitement et prodiguer des conseils sur la façon dont le praticien doit gérer l'alliance thérapeutique. Ces aspects renvoient au contenu mais également au processus du traitement.

Le G.L.M. nous oblige donc à nous questionner sur la posture à adopter lors d'un accompagnement avec une personne qui a contrevenu à certaines règles de la vie en société. En effet le processus de traitement impose à l'intervenant psychosocial de mobiliser des compétences théoriques mais aussi et surtout de développer des attitudes de disponibilité, d'ouverture, d'attention positive allant dans le sens d'un souci porté à la mobilisation des ressources de la personne.

Parler d'accompagnement dans une logique G.L.M. suppose de passer d'un modèle prescriptif à un modèle collaboratif.

Comme nous l'indique Paul (2010), le paradigme de l'accompagnement nous fait passer " d'une conception de la prise en charge dénoncée comme infantilisante et stigmatisante à une promotion des compétences sociales de la personne ». 
Le G.L.M. semble donc renvoyer à un contexte plus large que le R.B.R. L'objectif d'une approche centrée sur les forces permet d'aider le justiciable à obtenir les ressources psychologiques et sociales nécessaires à l'atteinte de buts qui ont une signification pour luimême. L'accompagnement au sens du G.L.M. et de Paul (2010) implique donc de passer par une notion de "changement expérimenté ici et maintenant, l'objectif étant de créer les conditions d'une relation qui permettent d'expérimenter le changement, de faire émerger la compétence à changer la situation ».

Les premières données de recherche indiquent que le G.L.M. est efficace pour surmonter certaines limites inhérentes aux approches traditionnelles de gestion du risque grâce, notamment, à l'amélioration de l'engagement dans le traitement, au soutien des mécanismes de désistement de la délinquance et à l'attention portée sur les contextes environnementaux (Ward, Yates et Willis, 2012).

Le G.L.M. ne prétend donc pas diminuer les risques de récidive mais bien favoriser la création de conditions internes et externes permettant de faciliter l'atteinte des besoins humains fondamentaux, conduisant à une vie plus satisfaisante que celle qui consistait à emprunter la voie du délit pour satisfaire des besoins non comblés.

\section{Les principes généraux du G.L.M. sont :}

Le délinquant sexuel est un être humain qui est animé par des besoins humains fondamentaux; comme tout autre être humain, il tente d'atteindre un bien-être psychologique via la satisfaction de certains « besoins primaires » (primary goods).

Dans cette logique, le délit sexuel n'est pas une fin en soi mais un moyen inadapté de parvenir à satisfaire des besoins humains fondamentaux.

Ward identifie 11 besoins primaires: la vie, le savoir, l'accomplissement dans les loisirs, l'accomplissement dans le travail, l'autonomie, l'équilibre émotionnel, l'amitié, la communauté, la spiritualité, le bonheur, la créativité.

Les besoins secondaires (secondary goods), quant à eux, constituent les moyens concrets d'accéder aux besoins primaires, ils prennent la forme de buts à atteindre ( $p$. ex. suivre une formation (besoin secondaire) peut satisfaire chez une personne plusieurs de ses besoins primaires comme le savoir, l'accomplissement dans le travail et l'amitié à travers de nouvelles rencontres.

\section{Les objectifs du G.L.M.}

$\checkmark$ Inciter à l'acquisition de ressources (internes et externes) ;

$\checkmark$ Réduire la récidive en tant qu'obstacle au bien-être, à l'accomplissement personnel et non en tant que cible unique et ultime du traitement.

Les implications thérapeutiques du G.L.M. (Ward, Mann et Gannon, 2007)

$\checkmark$ Pondération des besoins primaires : identification du poids accordé par le délinquant à chacun des besoins primaires permettant d'avoir une idée de ce que pourrait être pour lui une vie satisfaisante; 
Identification des objectifs et valeurs qui soutiennent la délinquance : quels sont les besoins que le délinquant sexuel tente de combler au travers de ses comportements délinquants?

$\checkmark$ Formulation des besoins secondaires : travail conjoint entre participant et clinicien afin d'identifier les façons socialement acceptables d'atteindre ses besoins primaires;

$\checkmark$ Élaboration du plan de vie : formulation du plan d'actions concrètes qui permettront au délinquant sexuel d'avoir accès à une vie plus épanouissante et selon des modalités non délinquantes.

\section{La notion centrale de disposition au traitement (Treatment Readiness)}

Nous l'avons déjà vu, le modèle de prévention de la rechute fondait l'efficacité des interventions sur le principe de consentement dans un premier temps et de motivation au traitement dans un second temps. Ce modèle s'appuyait largement sur le modèle transthéorique du changement et des quatre stades de changement (Prochaska et DiClemente, 1982). Dans cette optique, l'efficacité de l'intervention reposait sur les seules épaules du justiciable, la responsabilité de l'intervenant se limitant à le motiver.

Le concept, plus dynamique, de Disposition au traitement (Treatment Readiness) va apparaître pour nous livrer une vision plus globale et multidimensionnelle des conditions à envisager pour renforcer la disposition au traitement.

Sous l'impulsion de Ward avec son Modèle Multifactoriel de la Disposition au traitement des Délinquants (Ward et al., 2004) et de Burrowes et Needs, au travers de leur Cadre de Disposition au Traitement (Burrowes et Needs, 2009), le concept de Disposition au traitement va redéfinir de façon plus précise la nature du changement, mais également la place et la responsabilité tant de l'usager que de l'intervenant dans le processus de changement.

La Disposition au traitement est définie comme étant la présence de caractéristiques (états et dispositions) dans le chef du délinquant mais aussi de la situation thérapeutique qui sont susceptibles de promouvoir l'engagement dans la thérapie et donc l'amélioration des aptitudes au changement thérapeutique (Howell et Day, 2002, in Ward et al., 2004).

Le fait donc que le justiciable s'engage plus aisément et de façon plus impliquée dans une intervention dépendrait donc de facteurs qui lui sont propres, par exemple, sa motivation intrinsèque, mais également de facteurs propres aux caractéristiques du cadre d'intervention lui-même et, notamment, les conditions d'accessibilité au traitement proposé ou l'implication de l'intervenant dans celui-ci.

Ward et ses collaborateurs vont très précisément lister et développer l'ensemble des facteurs influençant la Disposition au traitement (Ward et al., 2004, traduit par nos soins).

\begin{tabular}{|l|l|}
\hline \multicolumn{1}{|c|}{ Facteurs internes } & \multicolumn{1}{c|}{ Facteurs externes } \\
\hline $\begin{array}{l}\text { Facteurs cognitifs: croyances, pensées et } \\
\text { aptitudes relatives à la capacité qu'a l'usager de } \\
\text { reconnaître qu'il a un problème, ses croyances } \\
\text { en l'efficacité d'une thérapie mais aussi ses }\end{array}$ & $\begin{array}{l}\text { Facteurs contextuels : incluant le fait de } \\
\text { déterminer si le traitement est légalement } \\
\text { contraint ou volontaire; }\end{array}$ \\
\hline
\end{tabular}


croyances à propos de ses propres ressources ;

Facteurs motivationnels tels qu'avoir la volonté de s'engager dans un traitement et avoir l'intention de poursuivre certains objectifs;

Facteurs comportementaux tels qu'avoir les compétences comportementales et intellectuelles pour pouvoir s'engager dans une thérapie;

Facteurs géographiques : incluant la distance séparant le lieu de dispense du traitement du lieu de résidence de l'usager;

Facteurs temporels : incluant que, tant du point de vue du client que des intervenants, suffisamment de temps sera disponible afin que le traitement puisse être mené à son terme;

Facteurs de ressources : incluant que l'équipe dispose non seulement des compétences suffisantes mais également d'un environnement physique adéquat (nombre de bureaux suffisants, salle d'attente...) pour mener à bien le traitement ;

Facteurs identitaires qui sont relatifs à la représentation que l'usager a de lui-même, à ses valeurs fondamentales et au type d'existence à laquelle il aspire.

\section{Facteurs liés aux caractéristiques du}

traitement: incluant le fait que les interventions soient conçues sur mesure en regard des besoins cliniques de l'usager mais également de ses préférences (intervention individuelle ou groupale);

Facteurs liés au support social: incluant le fait qu'un support interpersonnel puisse être apporté à l'usager durant le traitement (amis, famille, équipe d'intervention...).

Howells et Day (2003) précisent que la notion de disposition au traitement est plus inclusive que les termes de motivation et de réceptivité et qu'elle influe non seulement sur l'engagement dans le traitement mais aussi sur l'achèvement de celui-ci.

Pour promouvoir l'engagement, il est admis que le travailleur psychosocial, lui-même, s'implique dans l'intervention. En effet, la satisfaction du délinquant par rapport au traitement dépend de l'implication de l'intervenant (Levenson, Prescott et D'Amora, 2010).

Les études menées quant aux facteurs qui contribuent à la réussite des interventions de nature thérapeutique indiquent que tous les facteurs « concernent la qualité et la clarté de la relation (et très peu la maîtrise des techniques, quelles qu'elles soient...) » (Dorais, 2015).

Afin de susciter cet engagement, il est nécessaire de passer par une co-construction intervenant-délinquant du plan de traitement, impliquant ainsi une coresponsabilité, c'est-àdire un accord sur les buts, les objectifs et les besoins du patient et sur la définition du problème. L'intervenant psychosocial et le délinquant travaillent ensemble pour définir le problème présent et développer un plan de traitement. II s'agit donc d'une approche de personne à personne dans un environnement soutenant et non jugeant (Blagden et al., 2013) ainsi que non confrontant (Marshall et al., 2001), condition sine qua non pour permettre l'accessibilité au changement. Cette co-construction du processus de prise en charge suppose d'opérer un centrage sur la personne reconnue comme sujet actif de son parcours de vie et de son devenir. L'individu est donc vu comme unique "relié à son histoire, ses besoins, ses motivations, son désir, ses projets et dans sa globalité (son contexte, son milieu, son environnement, son réseau) »(Paul, 2010). 
Un autre facteur favorisant l'engagement du participant, dans l'approche G.L.M., est la responsabilité de l'intervenant d'adapter le traitement aux besoins des individus afin de les impliquer suffisamment pour qu'ils puissent accéder à un changement et aller jusqu'au bout dudit traitement. La responsabilité est donc bien partagée.

Le changement peut être considéré comme un processus riche, dynamique, influençable, canalisable et un phénomène non totalement prévisible sur lequel nous n'avons qu'un contrôle limité (Burrowes et Needs, 2009).

\section{Le G.L.M. comme invitation à un changement de posture chez l'intervenant psychosocial ?}

\section{En quoi les valeurs du travailleur psychosocial et celles du G.L.M. sont-elles compatibles?}

Au-delà de ce qui a pu être mis en évidence précédemment de ce qui, dans le G.L.M., outille l'intervenant psychosocial, autant dans le contenu que dans le processus d'accompagnement, le propos ici vise à articuler valeurs du travail social et principes du G.L.M. afin d'identifier en quoi le G.L.M., en s'inscrivant dans les valeurs du travail social, invite à un changement de posture professionnelle.

Le travail social poursuit comme but d'aider les individus à améliorer leur bien-être. « II s'engage à aider les individus à développer leurs habiletés et leur capacité d'utiliser leurs propres ressources et celles de la communauté pour résoudre leurs problèmes... » (définition ACTS).

Autant pour le G.L.M. que pour le travailleur psychosocial, nous sommes dans une perspective de travail qui promeut le bien-être des individus avant tout. II ne s'agit pas de se substituer à la personne mais bien de lui permettre de développer et d'utiliser ses habiletés, qu'elles soient personnelles ou sociales, en vue de la résolution de ses difficultés. Nous sommes donc bien loin de ce que nous pouvons parfois croiser dans nos trajectoires professionnelles, où une obédience peut s'imposer coûte que coûte, indépendamment des besoins de la personne, de ses spécificités et de ses aspirations.

\section{Le dénominateur commun entre (nouvelle) vision du travail social et G.L.M.}

Les valeurs d'humanité et de bien-être psychologique et social, défendues par le G.L.M., sont non sans lien avec les nouvelles visions du travail social. Par nouvelle vision nous entendons l'évolution des politiques sociales, notamment, qui ont invité les travailleurs œuvrant dans le social, à développer un point de vue plus systémique de la personne (passage de la personne perçue comme isolée de son contexte et carencée à une personne en relation avec son environnement et constitutive de ressources). En effet, comme nous le dit Astier (2007), cité par Franssen, Herscovici et Pierret (2008), le nouvel enjeu de la protection sociale est d'« équiper les individus pour qu'ils accèdent aux supports sociaux nécessaires à l'existence, qui leur permettent de s'activer, de s'autoréaliser en tant que personne mais sans continuellement payer de leur personne, c'est-à-dire sans être trop exposé à la responsabilisation de ce qui leur arrive ". La réalisation de soi permet d'obtenir la reconnaissance de son individualité et est donc intrinsèquement liée au social puisqu'on est individu relié au social et à l'environnement. Cette posture du social implique encore de passer « du travail sur autrui au travail avec autrui » (Franssen, Herscovici et Pierret, 2008). 
Le dénominateur commun entre travail social et G.L.M. est, sans ambiguïté, un but de prise en charge, quelle que soit la nature et l'objet de cette prise en charge (travail sous contrainte ou non), orienté vers la capacité du travailleur psychosocial à permettre à l'individu d'utiliser ses ressources pour s'autoréaliser, c'est-à-dire tendre à un état de bien-être. À ce titre, le G.L.M. propose, au travers de différents outils, de soutenir l'intervenant dans cette lourde tâche qu'est la poursuite du bien-être de la personne. À titre d'exemple, nous pouvons citer "l'Inventaire des souhaits et préoccupations personnels à destination des délinquant(e)s » adapté en langue française par Serge Corneille (Groupe Antigone, Université de Liège, Belgique, 2011), nommé en anglais Personal Aspirations and Concerns Inventory for Offenders (PACl-O) de Campbell, Sellen et McMurran, (2010). L'administration de l'Inventaire des souhaits et préoccupations se fait sous la forme d'un ou de plusieurs entretiens semi-structurés en face à face entre l'examinateur et le sujet. L'objectif est d'explorer six domaines de vie à l'aide de questions fournissant une base de discussion sur les préoccupations, les souhaits et les objectifs propres au délinquant. Ces six domaines de vie recouvrent les 11 besoins humains fondamentaux définis par Ward.

Le guide, composé de différentes questions dans différents domaines de vie de la personne, fournit une base de discussion, en particulier sur les préoccupations, les souhaits et les objectifs propres au sujet dans une logique singulière et globale à la fois.

Ainsi, dans l'approche G.L.M., on s'attelle à l'amélioration du bien-être et au développement d'une identité personnelle positive, en prenant en compte les caractéristiques de l'environnement dans lequel l'individu évolue. Il s'agit, comme déjà précisé, de le guider dans l'élaboration d'un plan de vie en prenant en compte ses besoins fondamentaux (primary goods) ainsi que les ressources et compétences nécessaires pour y arriver.

Cette philosophie, voire cette méthodologie, à penser et intervenir, contribue significativement à l'augmentation du sentiment de bien-être de l'individu et donc au renoncement à s'engager dans des conduites visant à dominer et à maîtriser l'autre.

De par le discours d'auteurs de violence sexuelle que nous avons en suivi actuellement, il apparaît que les intervenants psychosociaux témoignent d'une certaine frilosité à les prendre en charge et que, lorsqu'il y a prise en charge, celle-ci se centre exclusivement sur l'acte violent, sans prendre en considération la personne dans sa globalité.

Ces deux éléments conduisent bien souvent les auteurs à décrocher ou à se conformer uniquement dans la logique de répondre à l'injonction judiciaire, rendant ainsi le traitement inopérant et accroissant le risque de récidive. Le plan de traitement doit être adapté au participant, à ses capacités, à sa motivation. Le G.L.M. se centre sur les systèmes personnel, social et interpersonnel, mais aussi sur les dimensions comportementales et affectives de l'auteur.

La motivation dite extrinsèque, lorsque la mesure est imposée par le système judiciaire, peut conduire à une faible implication de l'auteur ; à l'inverse œuvrer à la construction d'un sentiment de vie plus épanouissante peut faciliter l'émergence d'une motivation dite intrinsèque et réduire le risque de récidive.

Le G.L.M. est un modèle d'intervention qu'on peut qualifier de global, cohérent et accessible à chaque intervenant, quelles que soient sa fonction, sa spécificité, sa formation. 
Dans l'optique G.L.M., se référer à un modèle global et cohérent de pensée et d'intervention (sans exclure les modèles classiques), en lien avec les besoins de la personne, assure une meilleure efficacité de la prise en charge.

\section{Illustration par une vignette clinique en contexte institutionnel}

En contexte institutionnel, un élément important à prendre en considération pour une prise en charge efficace et respectueuse de la personne est la vigilance de l'intervenant quant à une prise en charge «idéologique » débouchant sur des réunions d'équipe laissant bien souvent la place à des conflits idéologiques plutôt qu'au réel intérêt du patient.

Dans le tableau ci-dessous, nous avons tenté de conceptualiser, au départ d'une vignette clinique, un modèle de prise en charge se basant pour l'un sur une approche pluridisciplinaire n'intégrant pas l'approche G.L.M., pour l'autre, sur une approche G.L.M.

L'objectif ici n'est pas de faire une analyse de cas mais d'interroger le dispositif de prise en charge à la lumière de deux approches différentes et de souligner les écueils liés à une prise en charge non intégrée. La vignette clinique reprend la situation d'un jeune de 17 ans hospitalisé en unité de crise, au sein d'un hôpital psychiatrique, suite à la commission de faits d'inceste sur sa petite sœur. Son hospitalisation en psychiatrie est justifiée par la présence de troubles paranoïdes, nécessitant l'instauration d'un traitement à base de neuroleptiques. Le jeune est encadré par une équipe pluridisciplinaire, chacun des intervenants psycho-médico-sociaux disposant de formations et compétences propres.

Tableau comparatif entre l'approche traditionnelle et l'approche G.L.M.

\begin{tabular}{|c|c|}
\hline $\begin{array}{l}\text { Modèle de prise en charge traditionnel } \\
\text { pluridisciplinaire }\end{array}$ & Modèle de prise en charge G.L.M. \\
\hline $\begin{array}{l}\text { - Lectures croisées qui donnent lieu à des } \\
\text { interprétations différentes du comportement } \\
\text { du jeune } \\
\text { - Incohérence au niveau de la prise en charge } \\
\text { avec des directions de travail perçues } \\
\text { comme contradictoires par le patient } \\
\text { - L'équipe tire ses conclusions et livre son } \\
\text { diagnostic } \\
\text { - Prise en charge indifférenciée, gommage de } \\
\text { la richesse de la pluridisciplinarité en faveur } \\
\text { d'une lecture réductrice et non } \\
\text { responsabilisante des intervenants: " il } \\
\text { refuse le traitement, il est dans l'opposition, } \\
\text { on ne sait pas travailler avec lui » } \\
\text { Renforcement du besoin secondaire } \\
\text { (appartenance à un groupe de pairs } \\
\text { déviants) par non-comblement du besoin } \\
\text { primaire (besoin de reconnaissance et } \\
\text { d'existence) }\end{array}$ & 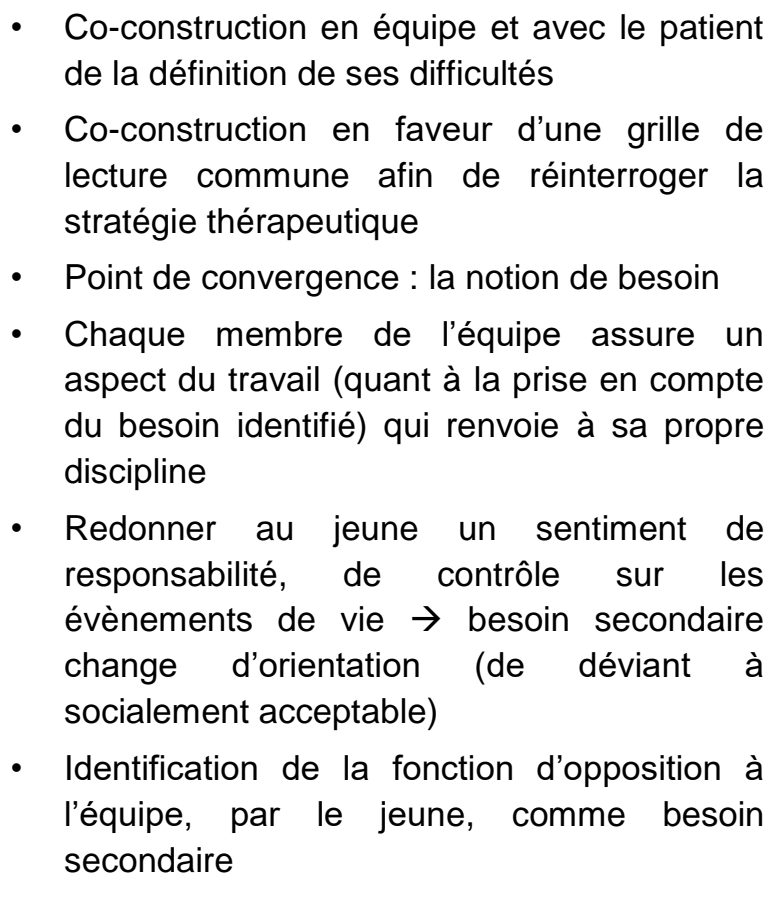 \\
\hline
\end{tabular}

Que peut-on retenir de ces deux approches? 
Le modèle traditionnel met l'accent sur l'expression des avis respectifs à chacune des fonctions dans l'équipe afin de livrer un savoir sur le patient. Les regards portés sur le patient apparaissent comme juxtaposés et non intégrés dans une vision globale de l'individu inscrit dans un contexte. Le besoin (secondaire) de s'affirmer dans des conduites anti-sociales (fréquentation de pairs délinquants, passages à l'acte hétéro-agressifs) est renforcé par l'attitude de l'équipe qui confronte le jeune à ses difficultés et le renvoie à une image négative (besoin primaire de reconnaissance et d'estime de soi) de lui-même.

L'approche G.L.M., quant à elle, situe chaque intervenant en complémentarité des rôles des autres professionnels de l'équipe. Chacun est respecté dans son rôle et dans sa fonction et s'inscrit en complémentarité du travail réalisé par ses collègues. Tant le justiciable que le travailleur psychosocial est réhabilité dans sa place.

Les termes de suivi, guidance, programmes de réadaptation, prise en charge, traitement ou injonction de soins sont généralement utilisés afin de désigner les obligations auxquelles le justiciable doit se soumettre dans le cadre d'une mise en liberté ou d'un maintien en liberté.

Pourtant, tous ces termes peuvent induire que le justiciable est objet de la mesure et peuvent mettre l'intervenant dans une position de dispensateur de services, d'expert d'un programme à administrer. Or, le G.L.M. impose à l'intervenant de resituer l'autre en position de sujet. Le processus est interactif et co-construit.

II se veut singulier puisqu'il est appelé à se réinventer à chaque rencontre. Barnao, Ward et Robertson (2015) parlent, d'ailleurs, d'une perspective constructiviste de la notion de réhabilitation dans le sens où la réalité est construite par et entre les personnes qui l'expérimentent. 
Schéma de la « posture de l'accompagnement »

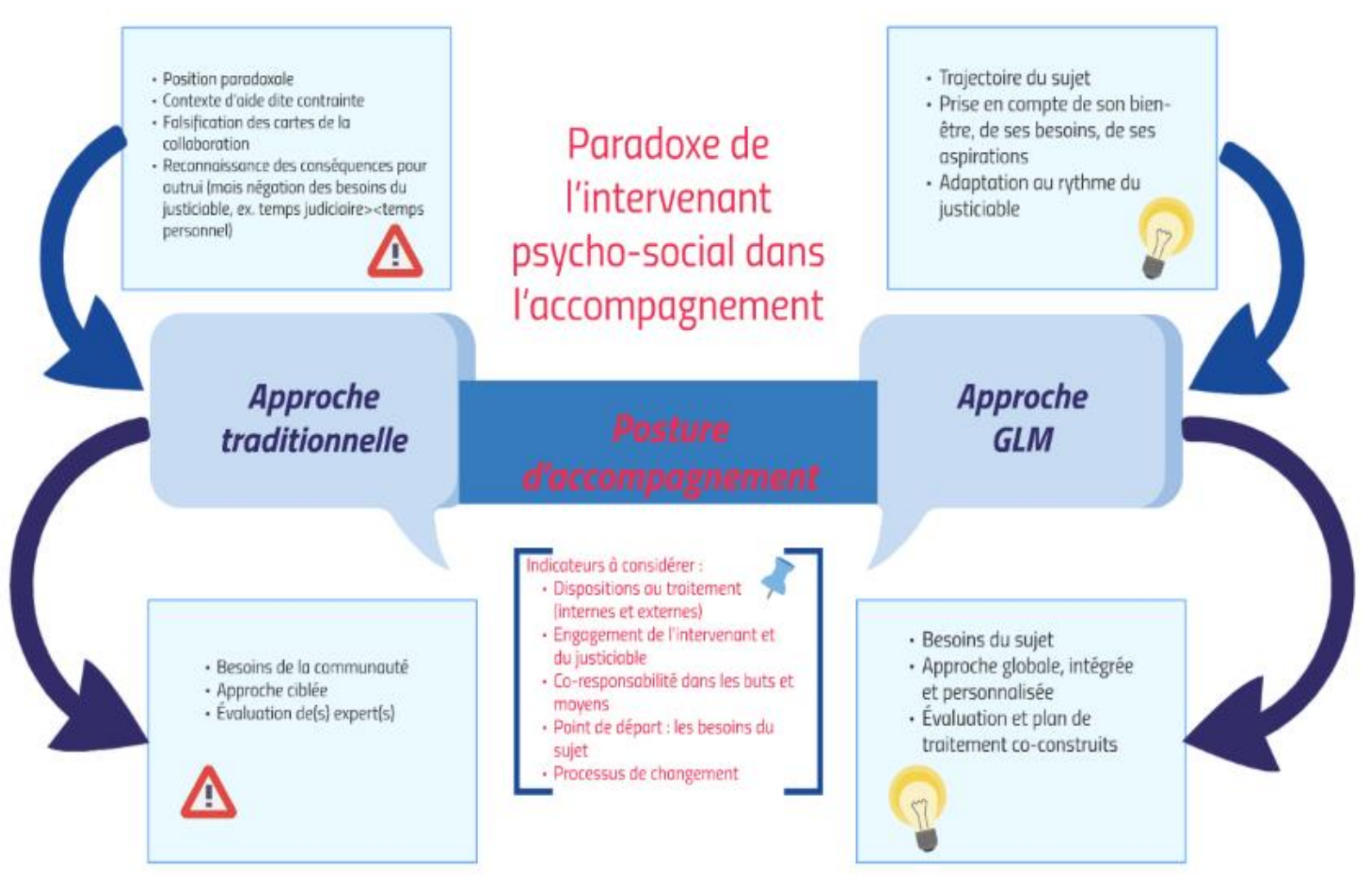

\section{Critiques et limites du G.L.M.}

De notre point de vue, la faiblesse majeure du G.L.M. réside dans l'ambiguïté que Ward entretient par rapport au concept de prévention de la récidive et qu'il présente, d'une part, comme un frein à l'épanouissement du délinquant (lequel vise en fait à la satisfaction de besoins humains fondamentaux et non de besoins sexuellement déviants) et, d'autre part, comme une des deux cibles d'une intervention G.L.M.

Nous pensons que, soit le risque de récidive est effectivement un frein à la satisfaction de besoins humains fondamentaux, auquel cas, la prévention de la récidive doit être envisagée comme un moyen parmi d'autres de travailler à cette satisfaction et non comme cible de l'intervention, soit la prévention de la récidive reste une des cibles du traitement et il faut, en ce cas, reconnaître que la récidive sexuellement délinquante est constitutionnelle du fonctionnement des délinquants sexuels.

Cette ambiguïté de Ward et de ses collègues amènera un certain nombre de critiques, parfois d'ailleurs antagonistes les unes des autres.

Ainsi, Glaser considérera que viser à la prévention de la récidive revient à maquiller une intervention punitive sous couvert d'une intervention socio-thérapeutique (Glaser, 2011) tandis qu'Andrews, Bonta et Wormith (2011) fustigeront l'a priori pro-social du G.L.M. Iorsque celui-ci affirme que le délinquant sexuel vise à la satisfaction de besoins humains non déviants.

D'autres critiques porteront, quant à elles, sur le peu de données probantes relatives aux interventions G.L.M. Certes, le G.L.M. est un modèle relativement récent mais quelques 
études ont déjà été menées à ce sujet (Simons et al., 2008, Harkins et al., 2012, Barnett et al., 2014). En conclusion, Hanson écrit en 2013: "Comme ce modèle est relativement récent, les recherches sur son efficacité n'en sont qu'à leur début. Cependant, les recherches préliminaires confirment les fondements théoriques de ce modèle et son impact positif sur les cibles du traitement telles qu'une motivation et une implication accrue dans le traitement, un taux d'abandon réduit et des progrès par rapport aux buts du traitement. » (Hanson et Yates, 2013).

Enfin, une étude très récente et non encore publiée de Pham et Saloppé (2016), présentée aux Premières journées internationales francophones du GLM à Tournai (Belgique), a permis d'établir une corrélation négative entre les résultats obtenus, d'une part, à des échelles actuarielles d'évaluation du risque de récidive et, d'autre part, les résultats obtenus à une échelle d'évaluation de la qualité de vie en général (whoqol), laquelle échelle recouvre en grande partie la liste des besoins humains fondamentaux établie par Ward. Ainsi, les justiciables bénéficiant d'une qualité de vie élevée (ou ayant satisfait en grande partie leurs besoins humains fondamentaux) étaient ceux qui présentaient le moindre risque de récidive, tandis que ceux bénéficiant d'une qualité de vie faible (ou n'ayant satisfait qu'à peu de leurs besoins humains fondamentaux) présentaient un risque de récidive accru. Les conclusions de cette étude semblent valider les fondements conceptuels du G.L.M.

\section{Conclusion}

La place du travailleur psychosocial dans l'intervention auprès de populations délinquantes, et notamment sexuellement délinquantes, est singulière dans la mesure où elle est contingentée non seulement par les cadres théorico-conceptuels auxquels le travailleur psychosocial va se référer mais surtout par des impératifs sociétaux de prévention de la récidive. Tout au long de cet article, nous avons voulu suggérer comment le travailleur psychosocial pouvait parvenir à concilier ses obligations éthiques d'aide à la personne et l'obligation qui lui est faite de veiller aux bénéfices de tiers en l'occurrence, les victimes et la société.

Réduits, dans le modèle de prévention de la récidive, à n'être que de simples agents de rééducation cognitivo-comportementale, dans une approche strictement standardisée et orthopédagogique, les travailleurs psychosociaux ont pu trouver dans les principes R.B.R. la possibilité de renouer avec une pratique plus nuancée, et ce, notamment, au travers du principe de réceptivité qui représentait une première ébauche d'individualisation des interventions.

L'avènement du G.L.M. permet aux travailleurs psychosociaux de retrouver leur place d'aidant dans une approche plus globale et plus contextualisée, se fondant, de surcroît, bien plus sur les ressources que sur les carences de l'individu.

Avec le G.L.M., nous avons apporté des arguments en faveur d'une posture de l'intervenant psychosocial qui rompe avec les standards classiques de prévention de la récidive et qui permette à l'intervenant de se situer dans une réelle logique d'humanisation de l'usager et d'individualisation de l'intervention. La conception même du justiciable comme être de besoin et non pas comme être carencé nous invite à dégager les voies propices à la mobilisation des ressources de la personne. Ce postulat permet de se distancier des étiquettes stigmatisantes et d'opérer une distinction entre la personne et son comportement. Nous avons remis l'accent, comme d'autres auteurs l'ont fait avant nous, sur l'importance capitale des attitudes dans l'accompagnement, attitudes qui passent par une compréhension des 
comportements humains lus en tant que conduites humaines visant prioritairement à satisfaire des besoins humains (Dorais, 2015).

Enfin, le G.L.M. nous a rappelé ce qui ne devrait jamais s'oublier à savoir que c'est aux intervenants à s'adapter aux besoins de chaque usager, en inventant chaque fois des réponses singulières, et non pas à l'usager d'adapter ses besoins au modèle de référence de l'intervenant et aux compétences limitées de ce dernier.

Enfin, si nous désirons dépasser les limites du G.L.M. et les débats (souvent stériles et de fort peu d'intérêt) qui l'opposent aux principes du R.B.R., nous suggérons de faire du G.L.M. une lecture hétérodoxe.

En effet, nous plaidons pour un G.L.M. dans lequel, assurément, la prévention de la récidive ne constituerait qu'un moyen (parmi de nombreux autres) de parvenir à atteindre la cible unique et ultime de l'intervention que constitue la satisfaction des besoins humains fondamentaux de la personne. De cette façon, lorsque serait abordé avec l'usager la nécessité (pour son propre bénéfice et non plus au bénéfice d'un tiers) de prévenir une éventuelle récidive, nous recommanderions, bien évidemment, d'appliquer les principes R.B.R. mais uniquement, à ce stade de l'intervention et sachant que cette dernière ne peut se réduire au travail sur la prévention de la récidive.

On nous objectera, probablement, que, au travers de cette lecture hétérodoxe, nous dénaturons les fondements mêmes du G.L.M. et ce reproche sera parfaitement légitime. Mais nous pensons que l'intérêt des modèles est de permettre aux acteurs de terrain de les déformer et de les adapter à leur cadre de travail, bien plus que de constituer un cadre rigide et contraignant.

De notre point de vue un modèle de réhabilitation quel qu'il soit, doit constituer une source d'inspiration, de liberté, de créativité et d'efficacité au bénéfice du plus grand nombre, plutôt qu'un carcan dogmatique entravant les capacités d'action et de réflexion des intervenants.

\section{Serge Corneille \\ Bérengère Devillers}

Groupe Antigone, Université de Liège, Belgique

\section{RÉFÉRENCES}

ACTS (Association canadienne des travailleurs sociaux). Le travail social, qu'est-ce que c'est? Consulté le 12 décembre 2015 au <http://www.casw-acts.ca/fr/le-travail-socialquest-ce-que-cest>.

Andrews, D.A., J. Bonta et J.S. Wormith (2011). "The Risk-Need-Responsivity (RNR) model : Does Adding the Good Lives Model contribute to Effective Crime Prevention ? ", Criminal Justice and Behavior, vol. 38, n 7, p. 735-755.

Barnao, M., P. Robertson et T. Ward (2010). « The Good Lives Model applied to a forensic population », Psychiatry, Psychology and Law, vol. 17, n 2, p. 202-217.

Barnao, M., T. Ward et P. Robertson (2015). «The Good Lives Model: A new paradigm for forensic mental health », Psychiatry, Psychology and Law, vol. 23, $n^{\circ} 2$, p. 288-301. 
Barnett G.D., Mandeville-Norden R. \& Rakestrow J. (2014). The Good Lives Model or Relapse Prevention: What Works Better in Facilitating Change? Sexual abuse: A Journal of Research and Treatment, 26 (1), 3-33.

Blagden, N., B. Winder, M. Gregson et K. Thorne (2013). «Working with denial in convicted sexual offenders: A qualitative analysis of treatment professionnals' views and experiences and their implications for practice ", International Journal of Offender Therapy and Comparative Criminology, vol. 57, n 3, p. 332-356.

Burrowes, N. et A. Needs (2009). "Time to Contemplate Change?: A Framework for Assessing Readiness to Change with Offenders ", Aggression and Violent Behavior, vol. $14, n^{\circ} 1$, p. 39-49.

Campbell, J., J.L. Sellen et M. McMurran (2010). "Personal Aspirations and Concerns Inventory for Offenders: Developments in the measurement of offenders's motivation », Criminal Behavior and Mental Health, vol. 20, $\mathrm{n}^{\circ}$ 2, p. 144-157.

Corneille, S., et N. Henrard (2017). "Le Good Lives Model : un modèle alternatif au modèle de prévention de la récidive ", in E. Dieu (dir.). Les innovations criminologiques, Paris, L'Harmattan, p.89-109. (sous presse).

Dieu, E., (2017). "PRATIC-SERA" ou le "Parcours initiatique pour savoir qui je suis", l'accompagnement positif en groupe des PPSMJ. In E. Dieu, "Les innovations criminologiques", L'Harmattan.

Dorais, M. (2015). Le métier d'aider, Montréal, VLB éditeur.

Franssen, A., A. Herscovici et J. Pieret (2008)., « Autonomisation et responsabilisation des individus: qu'est-ce qui ne va pas? », Bruxelles informations sociales, $\mathrm{n}^{\circ} 159$, «L'individualisation des politiques sociales ».

Franssen, A. (2015). “Travailleurs sociaux : identités en question », conférence présentée à l'École supérieure d'action sociale Haute École libre mosane, Liège, Belgique.

Hanson, R.K., et K.E. Morton-Bourgon (2005). "The characteristics of persistent sexual offenders: A meta-analysis of recidivism studies ", Journal of Consulting and Clinical Psychology, vol. 73, $\mathrm{n}^{\circ}$ 6, p. 1154-1163.

Hanson, R.K. \& Yates, P.M. (2013). Psychological treatment of sex offenders. Current Psychiatry Reports, 15(3), 348.

Harkins, L., Flak, V., Beech, A., Woodhams, J. (2012). Evaluation of a Community-Based Sex Offender Treatment Program Using a Good Lives Model Approach . Sexual Abuse, $\mathrm{XX}(\mathrm{X}), 1-27$.

Howells, K., et A. Day (2003). «Readiness for anger management: Clinical and theoretical Issues », Clinical Psychology Review, vol. 23, no 2, p. 319-337.

Howells K, (2008). « Readiness for treatment in forensic populations », Rotterdam. 
Langlands, R.L. de, T. Ward et E. Gilchrist (2009). « Applying the Good Lives Model to Male Perpetrators of Domestic Violence », Behaviour Change, vol. 26, n² 2, p. 113-129.

Levenson, J.S., D.S. Prescott et D.A. D’Amora (2010). « Sex offender treatment : consumer satisfaction and engagement in therapy ", International Journal of Offender Therapy and Comparative Criminology, vol. 54, $\mathrm{n}^{\circ} 3$, p. 307-326.

Marshall, W.L., D. Thornton, L.E. Marshall, Y.M. Fernandez et R. Mann (2001). «Treatment of sexual offenders who are in categorical denial: A pilot project ", Sexual Abuse: $A$ Journal of Research and Treatment, vol. 13, n 3, p. 205-215.

Paul, M. (2010). "L'accompagnement: une posture professionnelle spécifique », Les carnets de la Persagotière, $\mathrm{n}^{\circ} 22$, p. 1-21.

Pham, Th. et Saloppé, X. (2016, octobre). Qualité De Vie (QDV) : Quel apport pour le Good Lives Model (GLM) ? Communication dans le cadre de la Plénière N8, Good Lives Model (GLM): Premières rencontres internationales francophones, CRP Les Marronniers, Tournai - Belgique.

Pithers, W.D., J.K. Marques, C.C. Gibat et G.A. Marlatt (1983). «Relapse prevention: A selfcontrol model of treatment and maintenance of change for sexual aggressives ", in J.G. Greer et I.R. Stuart (dir.), The sexual aggressor: Current perspective on treatment, New York, Van Nostrand Reinhold.

Print, B. (dir.) (2013). The Good Lives Model for Adolescents Who Sexually Harm, Brandon (VT), Safer Society Press.

Prochaska, J.O et Di Clemente, C.C. (1982). "Transtheoretical-therapy : Toward a more integrative model of change. Psychotherapy: Theory, Research, and Practice, 19, 276288

Sellen, J.L., M. McMurran, W.M. Cox, E. Theodosi et E. Klinger (2006). «The Personal Concerns Inventory (Offender Adpatation): Measuring and enhancing motivation to change ", International Journal of Offender Therapy and Comparative Criminology, vol. $50, n^{\circ} 3$, p. 294-305.

Simons, D., McCullar, B., et Tyler, C. (2008). Evaluation of the Good Lives Model approach to treatment planning. Presented at the 27th annual research and treatment conference of the Association for the Treatment of Sexual Abusers, Atlanta, GA.

Vanderstukken, O., et M. Benbouriche (2013). "Les interventions cognitivocomportementales destinées aux auteurs d'agression sexuelle », Poitiers.

Ward, T., A. Day, K. Howells et A. Birgden (2004). «The multifactor offender readiness model », Aggression and Violent Behavior, vol. 9, n 6, p. 645-673.

Ward, T., et A. Beech (2006). " An integrated theory of sexual offending », Aggression and Violent Behavior, vol. 11, n 1, p. 44-63. 
Ward, T., R. Mann et T.A. Gannon (2007). «The Good Lives Model of rehabilitation: Clinical implications », Aggression and Violent Behavior, vol. 12, n 1, p. 87-107.

Ward, T., et S. Maruna (2007). Rehabilitation: Beyond the risk assessment paradigm, Londres, Routledge.

Ward, T., P.M. Yates et G.M. Willis (2012). "The Good Lives and the Risk Need Responsivity model: a critical response to Andrews, Bonta \& Wormith (2011) », Criminal Justice and Behavior, vol. 39, no 1, p. 94-110. 